\title{
Variación en la composición isotópica del agua meteórica a lo largo de la sección centro-noreste de la Sierra Madre Oriental
}

César F. Aguilar-Ramírez, Antoni Camprubí, Elisa Fitz-Díaz, Edith Gienfuegos-Alvarado, Pedro Morales-Puente

César F. Aguilar-Ramírez

aguilar.ce.fe@gmail.com

Programa de Posgrado en Ciencias de la Tierra, Universidad Nacional Autónoma de México; Ciudad Universitaria, 04510 Coyoacán, CDMX, México.

\section{Antoni Camprubí \\ Elisa Fitz Díaz \\ Edith Gienfuegos Alvarado \\ Pedro Morales Puente}

Instituto de Geología, Universidad Nacional Autónoma de México; Ciudad Universitaria, 04510 Coyoacán, CDMX, México

BOL. SOC. GEOL. MEX. 2017

VOL. 69 NO. 2

P. $447-463$

Manuscrito recibido: Agosto 8, 2016. Manuscrito corregido: Noviembre 2, 2016. Manuscrito aceptado: Diciembre 15, 2016

\section{RESUMEN}

Se analizan las variaciones en la composición isotópica de agua meteórica a lo largo de una sección en el Cinturón de Pliegues y Cabalgaduras Mexicano, ubicado en la sección centro-noreste de la Sierra Madre Oriental. El objetivo de ello es determinar dichas variaciones en función de:1) el incremento en la elevación topográfica y 2) la distancia de las precipitaciones al interior del continente, desde que se produjo la evaporación de agua oceánica del Golfo de México. Se consideraron las características topográficas y climáticas de la sección estudiada, seleccionada por presentar las ventajas detalladas como sigue. La deformación en esta sección generó un rasgo de cuña orogénica, que le confiere una pendiente promedio entre $1^{\circ}$ y $1.5^{\circ}$, sin la presencia de barreras topográficas que dificulten la libre circulación de vientos. Ello favorece evaluar variaciones significativas en la composición isotópica de $\mathrm{H}$ y $\mathrm{O}$ en función de la elevación topográfica. La sección se ubica a una latitud prácticamente constante entre $21^{\circ}$ y $22^{\circ} \mathrm{N}$, y la temperatura media anual decrece gradualmente desde el antepaís hacia el transpaís de la sección durante los periodos de precipitación. El muestreo de agua meteórica se efectuó mediante la colocación de 12 colectores de agua de lluvia en lugares elevados, a alturas entre 2740 y $56 \mathrm{msnm}$ a lo largo de la sección estudiada. Asimismo, se tomaron muestras de agua de ríos y manantiales durante la temporada de lluvia de 2012 en zonas de escorrentía constante, de 7 localidades diferentes. Los valores obtenidos en agua de lluvia varían entre -4.89 y $-11.75 \%$ para $\delta^{18} \mathrm{O}$ y entre -25.35 y $-80.34 \%$ para $\delta^{2} \mathrm{H}$ (respecto a VSMOW) Así, se determinó una Línea de Agua Meteórica local (LAML) definida por la ecuación $\delta^{2} \mathrm{H}$ $=\delta^{18} \mathrm{O} * 8.15+15$. La tasa de fraccionamiento obtenida para $\mathrm{O}$ y $\mathrm{H}\left(\delta^{18} \mathrm{O}\right.$ y $\left.\delta^{2} \mathrm{H}\right)$ respecto a la elevación, fue de $2.19 \% \mathrm{~km}^{-1}$ y $17.75 \% \mathrm{~km}^{-1}$, respectivamente. La LAML y las tasas de fraccionamiento obtenidos en este estudio podrán ser utilizados como valores de referencia a nivel regional para evaluar el papel del agua meteórica involucrada en procesos atmosféricos y corticales superficiales.

Palabras clave: Composición isotópica, $\delta^{2} \mathbf{H}, \delta^{18} \mathbf{O}$, agua meteórica, fraccionamiento isotópico, isótopos estables.

\section{ABSTRACT}

We analyze the variations in isotopic composition of meteoric water along a transect in the Mexican FoldThrust Belt, located in the central-northeastern part of the Sierra Madre Oriental. The aim is to determine such variations as a function of 1) increasing topographic elevation and 2) the distance of rain inside the continent since evaporation occurrence of oceanic water from the Gulf of Mexico. Topograph$i c$ and climate characteristics were considered in the studied section, which was chosen because it presents some advantages as follows. Deformation in this section exhibits orogenic wedge features, which generated an average slope between $1^{\circ}$ and $1.5^{\circ}$, with no topographic barriers that obstruct the free circulation of wind. This allows us to evaluate significant isotopic variations of $H$ and $O$ as a function of topographic elevation. The section is located at constant latitude between $21^{\circ}$ and $22^{\circ} \mathcal{N}$. Furthermore, the average annual temperature decreases gradually from the foreland to the hinterland of the section during precipitation periods. Sampling of meteoric water was carried out by installing 12 rainwater collectors at heights between 2740 and 56 masl along the transect. Additionally, water samples were collected in rivers and springs during the 2012 rain season in zones of constant water runoff in 7 different localities. Obtained values from rain water vary between -4.89 and $-11.75 \%$ for $\delta^{18} \mathrm{O}$ and between -25.35 and $-80.34 \%$ for $\delta^{2} \mathrm{H}$ (relative to VSMOW)These allowed to determine a Local Meteoric Water Line (LMWL) defined by the equation $\delta^{2} \mathrm{H}=\delta^{18} \mathrm{O}$ * $8.15+15$. With this, fractionation rate relative to elevation for $\mathrm{O}$ and $\mathrm{H}\left(\delta^{18} \mathrm{O} y \delta^{2} H\right)$ were obtained at $2.19 \% \mathrm{~km}^{-1}$ and $17.75 \% \mathrm{~km}^{-1}$, respectively. The LMWL and fractionation rates obtained in this study can be used as regional-scale references in order to evaluate the role of meteoric water involved in atmospheric and shallow cortical processes.

Keywords: Isotopic composition, $\delta^{2} H$, $\delta^{18} \mathrm{O}$, meteoric water, isotopic fractionation, stable isotopes. 


\section{Introducción}

El agua meteórica reside en la superficie continental en forma de glaciares, mantos acuíferos, ríos y lagos. Se origina por la descarga de nubes en forma de lluvia, granizo o nieve al interior de los continentes (Mook, 2002; Sharp, 2007). El 90 \% de la precipitación pluvial en la Tierra se produce sobre los océanos, y tan solo un $\sim 10 \%$ se moviliza en masas de aire al interior de los continentes.

Esta fuente de agua genérica no posee una composición isotópica fija, pues se modifica en su distribución a escala global mediante procesos de fraccionamiento isotópico (Craig, 1961) que controlan la composición isotópica en la precipitación de una masa dada, principalmente en la fase de vapor remanente que queda en dicha masa de aire (Poage y Chamberlain, 2001; Mook, 2002; Sharp, 2007). El fraccionamiento isotópico se inicia con la evaporación de agua oceánica (la principal fuente de agua meteórica), de forma que las moléculas de agua evaporada incorporan preferentemente los isótopos más ligeros de $\mathrm{O}$ y $\mathrm{H}$ $\left({ }^{1} \mathrm{H}\right.$ y $\left.{ }^{16} \mathrm{O}\right)$. Por el contrario, las moléculas de agua constituidas por átomos más pesados de $\mathrm{O}$ y $\mathrm{H}\left({ }^{2} \mathrm{H}\right.$ $\left.\mathrm{y}{ }^{18} \mathrm{O}\right)$ se fraccionan principalmente hacia la fase líquida (Dansgaard, 1964). Los principales factores de fraccionamiento isotópico que controlan las variaciones en la concentración de isótopos estables de $\mathrm{H}$ y $\mathrm{O}$ en el agua meteórica son:

1. La temperatura: cuando la radiación solar incide sobre la superficie oceánica genera evaporación en la superficie de la misma, generando un cambio de fase que propicia fraccionamiento isotópico en las moléculas de agua. El fraccionamiento isotópico producido por las variaciones en la temperatura obedece a que la energía de vibración de las moléculas de agua es mayor a medida que incrementa la temperatura, propiciando la incorporación de las moléculas más pesadas hacia la fase vapor (Mook, 2002).

2. Latitud: está relacionada con el grado de radiación solar sobre la superficie oceánica. La composición isotópica disminuye con el incre- mento en la latitud, de forma que a mayores latitudes el agua en la fase de vapor será isotópicamente más ligera que en zonas ecuatoriales. Tal fenómeno es debido esencialmente a la disminución de la temperatura con la latitud, de forma que en zonas ecuatoriales tiende a atenuarse el fraccionamiento entre el isótopo ligero y el pesado de un elemento, pudiéndose incorporar una mayor cantidad relativa del isótopo pesado a la fase vapor (Craig, 1961; Poage y Chamberlain, 2001; Mook, 2002, Sharp, 2007).

3. Distancia que recorren las nubes al internarse al continente: asociado dinámicamente a los cambios en la temperatura atmosférica y a las barreras topográficas con las que se encuentre configurado el relieve. Las variaciones en la temperatura atmosférica generan el punto de rocío y, consigo, las precipitaciones que dan lugar a cuerpos de agua meteórica en la superficie. Por su parte, las barreras topográficas impiden la descarga homogénea de agua a lo largo de las cordilleras (Craig, 1961; Poage y Chamberlain, 2001; Mook, 2002, Sharp, 2007).

4. Elevación topográfica: se registran composiciones isotópicas menores a medida que se incrementa la elevación. De acuerdo con Mook (2002), este efecto se relaciona con la temperatura, debido a que la condensación se produce a partir de la disminución de la misma con el aumento de la altitud. Al disminuir la presión con el incremento en la altitud, el sistema requiere un descenso de la temperatura mayor que en el caso de la condensación por presión isobárica para poder alcanzar la presión de vapor de agua saturada (Dansgaard, 1964; Molnar, 2010). El fraccionamiento isotópico debido a variaciones topográficas tiene lugar en las cordilleras montañosas cuando las nubes viajan al interior de los continentes a lo largo de una pendiente topográfica en incremento: una parte de la fase vapor se condensa y precipita a determinada altura al llegar al punto de rocío. Esto continúa produciéndose a medida 
que la elevación topográfica se incrementa en su recorrido por la cordillera (Ambach et al., 1968; Rowley y Grazione, 2007; Sharp, 2007; Campani et al., 2012; Poulsen y Jeffery, 2012), en el mismo proceso de fraccionamiento isotópico. De tal forma, las moléculas de agua constituidas por los isótopos ligeros de $\mathrm{O}$ y $\mathrm{H}$ permanecerán preferentemente en la fase vapor respecto a la fase líquida que precipita a partir de ella. A partir de precipitaciones sucesivas, el agua de lluvia devendrá isotópicamente más ligera con cada precipitación; dicho proceso se conoce como "destilación de tipo Rayleigh".

5. Efecto de la orografía: parte de la problemática que diversos autores han encontrado al realizar estudios sobre las variaciones isotópicas en agua meteórica radica en la configuración de barreras en el relieve que obstruyen el libre paso del vapor atmosférico en las cordilleras. Estas provocan un fraccionamiento isotópico dinámico al canalizar y concentrar el vapor atmosférico, y forzando con ello la descarga de nubes hacia los valles conformados de manera local.

6. Efecto de pseudoaltitud: consiste en un enriquecimiento en ${ }^{18} \mathrm{O}$ y ${ }^{2} \mathrm{H}$ durante la evaporación de las gotas de lluvia por debajo de la base de las nubes, principalmente en las zonas de poca altitud, tales como valles profundos en zonas montañosas (Moser y Stichler, 1974; Schemmel et al., 2013).

El estudio de la composición isotópica de agua meteórica se ha aplicado en los últimos años con especial énfasis para determinar la paleoaltimetría de algunas cordilleras orogénicas (Chamberlain et al., 1999; Garzione et al., 2000; Rowley 2007; Rowley y Grazione, 2007; Campani et al., 2012). Por ello, la necesidad de entender con mayor detalle los factores que modifican la composición isotópica del agua meteórica que descarga en los continentes ha adquirido una importancia especial. De ahí parte la necesidad de estudios relacionados con la elevación topográfica y la distancia que recorren las nubes a lo largo de la pendiente topográfica hasta encontrar barreras topográficas que puedan promover la generación de precipitaciones. Hasta el momento, sólo existe un estudio sistemático en México enfocado en determinar la línea de agua meteórica local en asociación con un orógeno (Pérez Quezadas et al., 2015). Los estudios existentes sobre la composición isotópica del agua meteórica son de escaso alcance, y están mayoritariamente relacionados con estudios de origen del agua en campos geotérmicos (e.g., Truesdell et al., 1978; Tello, 1982; González-Partida et al., 2005; Birkle et al., 2016).

En el presente estudio se analizan las variaciones en la composición isotópica de agua meteórica en una sección del Cinturón de Pliegues y Cabalgaduras Mexicano (CPCM), a fin de obtener sólo las variaciones debidas al incremento en la elevación topográfica. Se trata de establecer la línea del agua meteórica local (LAML) a partir del análisis de las concentraciones isotópicas de agua de lluvia, con la finalidad de fijarla como grupo de valores de referencia que permita discriminar entre fuentes de agua distintas en cualquier estudio isotópico de $\mathrm{O}$ y H. La LAML permitirá identificar la presencia de agua meteórica en estudios sobre geoquímica de fluidos en yacimientos minerales, geología estructural, hidrogeología, etc. La sección del CPCM estudiada se ubica geográficamente desde el centro del país hacia el noreste, hasta las inmediaciones de la Planicie Costera del Golfo de México (Figura 1). Por sus características morfológicas y ubicación geográfica, el CPCM en su parte central constituye un laboratorio natural para este tipo de estudio.

\section{Características de la región estudiada}

\subsection{GEOLOGÍA}

El Cinturón de Pliegues y Cabalgaduras Mexicano (CPCM) es un rasgo orogénico en forma de cuña que se emplazó por deformación de piel delgada thin-skinned, producto de la exhumación y acortamiento de cuatro elementos paleo- 


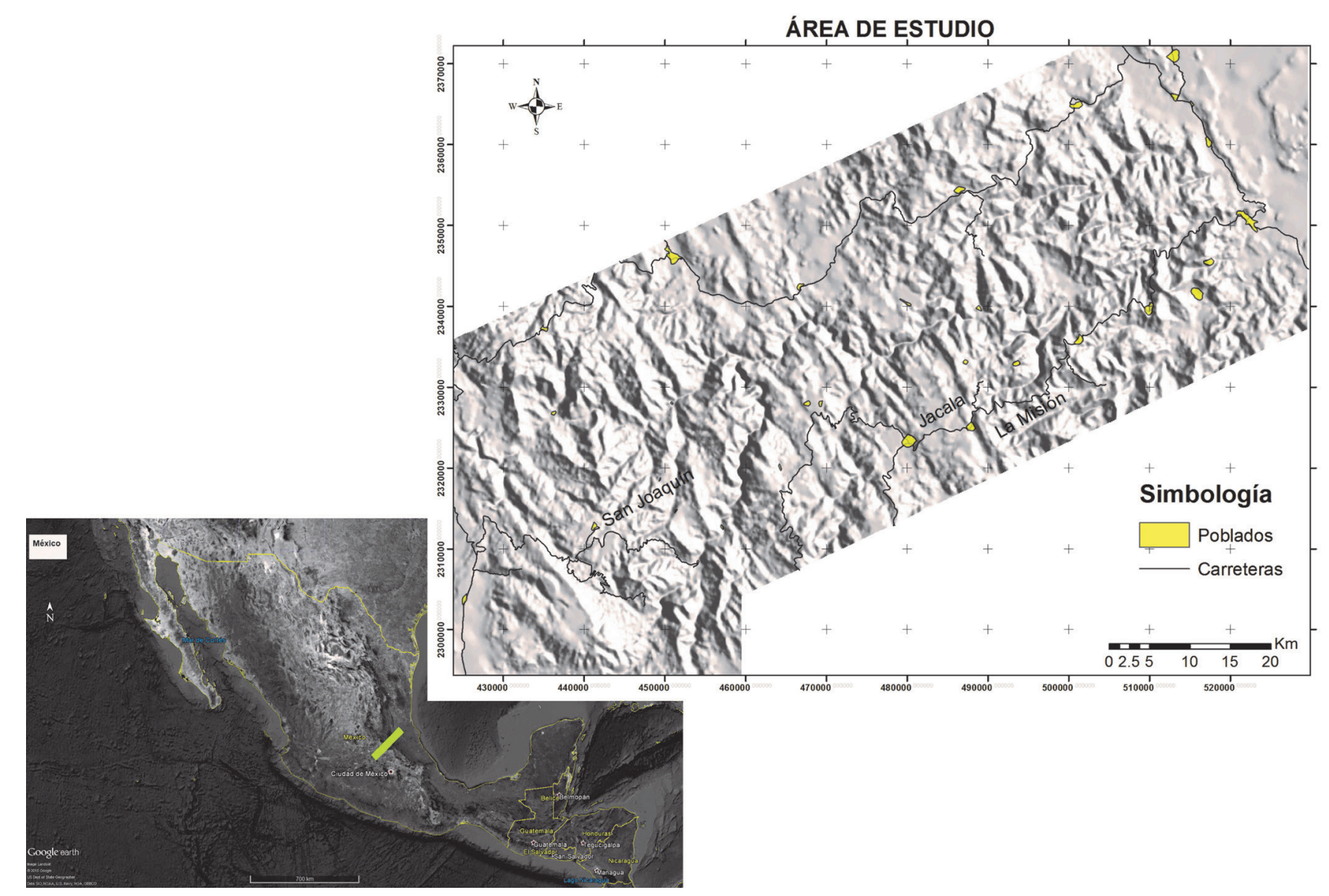

Figura 1 Mapa de ubicación de la zona de estudio, con una longitud de poco más de $125 \mathrm{~km}$.

geográficos (Suter, 1980, 1984, 1987; Eguiluz de Antuñano et al., 2000; Fitz-Díaz, 2010; Fitz-Díaz et al., 2011). Tales elementos son dos plataformas marinas (El Doctor y Valles-San Luis Potosí) y dos cuencas sedimentarias (Zimapán y Tampico-Misantla; figuras 2 y 3). Éstos corresponden a la configuración predeposicional del basamento en pilares y fosas, respectivamente, generadas durante el proceso de apertura del Golfo de México. En las rocas de la Plataforma El Doctor se desarrollaron principalmente cabalgaduras. La parte oriental de dicha plataforma cabalga sobre rocas de la Cuenca de Zimapán, donde la deformación propició el emplazamiento de anticlinales y sinclinales asimétricos con sus flancos frontales invertidos, inclinados hacia el suroeste (Carrillo, 1971; Carrillo-Martínez, 1990). El borde oriental de la
Cuenca de Zimapán cabalga, a su vez, sobre la Plataforma Valles-San Luis Potosí, la cual muestra deformación similar a la de la Plataforma El Doctor. Asimismo, la Plataforma Valles-San Luis Potosí cabalga en su borde oriental sobre la Cuenca Tampico-Misantla, que presenta desarrollo de pliegues transportados en dirección ENE. Es decir, las cuencas y plataformas se invirtieron, pasando a ser elementos topográficos positivos, en forma de escamas tectónicas imbricadas hacia el oriente, y registran una propagación en la intensidad de la deformación con esfuerzos orientados de SW a NE (Campa-Uranga, 1983; Davis et al., 1983; Eguiluz de Antuñano et al., 2000; Fitz-Díaz et al., 2010, 2011, 2014; Ortega-Flores, 2011; ver Figura $3)$. 


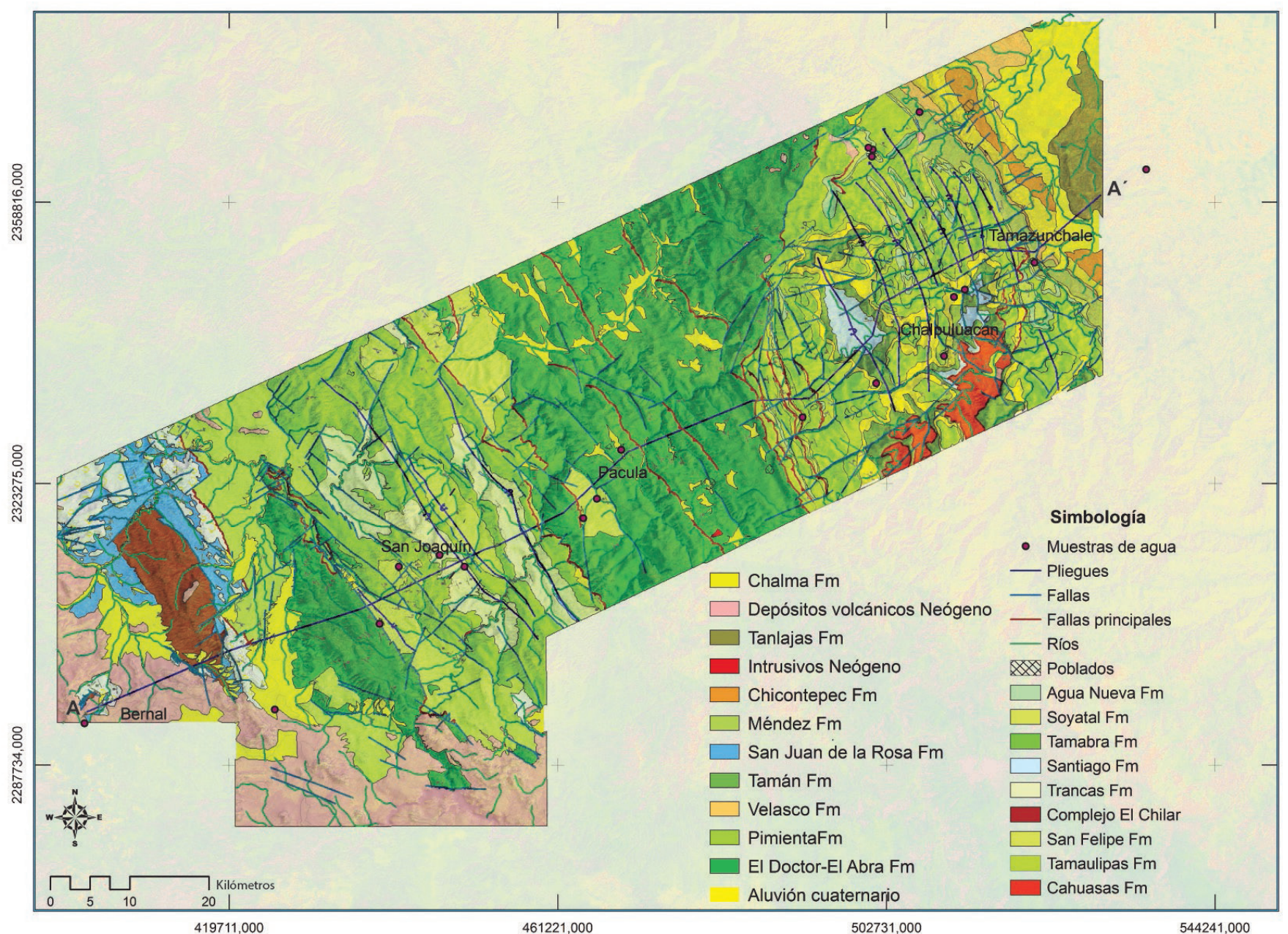

Figura 2 Mapa geológico de la región estudiada. La sección estudiada va de A hacia A', en círculos rojos se indican las localidades de muestreo. Modificado de Fitz-Díaz et al. (2011).

SW

NE

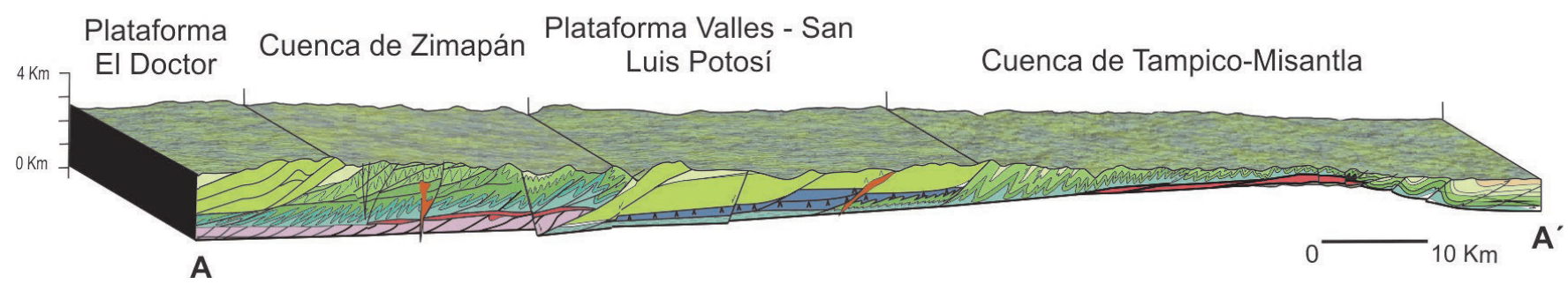

Figura 3 Perfil geológico de la sección estudiada, que muestra la disposición espacial de los elementos paleogeográficos que la componen, la geometría de la cuña orogénica en función de los mismos y una representación del relieve con las elevaciones topográficas máximas y mínimas. Modificado de Fitz-Díaz et al. (2011). 


\subsection{GEOMORFOLOGÍA Y GLIMA}

La sección de muestreo se ubica en el centro del país y se extiende hacia el noreste del país atravesando los estados de Querétaro, Hidalgo y San Luis Potosí. El conjunto de características morfoclimáticas que la caracterizan son:

1. Se ubica en una latitud prácticamente fija entre $21^{\circ}$ y $22^{\circ} \mathrm{N}$, por lo que no se esperan variaciones en la composición isotópicas de agua meteórica debidas al efecto latitudinal.

2. Presenta un relieve en forma de cuña orogénica con una pendiente continua entre $1^{\circ}$ y $1.5^{\circ}$ (Fitz-Díaz et al., 2011), con elevaciones máximas de aproximadamente $3100 \mathrm{msnm}$, descendiendo hasta la Planicie Costera del Golfo de México (Figura 4), y sólo existen algunos valles y depresiones del orden de pocas decenas de metros. Tales características permiten obviar el efecto de pseudoaltitud.

3. No existen fuentes relevantes adicionales de agua meteórica que puedan modificar significativamente la composición isotópica de agua precipitada por el acarreo de nubes desde el Golfo de México.

4. No existen barreras topográficas que impidan la libre circulación de los vientos alisios, lo cuales son responsables del transporte de nubes que descargan al interior del continente. Ello permitió la captación del agua meteórica derivada de cada destilación de tipo Rayleigh producida a consecuencia de la continentalización de las masas de vapor de agua.

5. Los vientos que transportan el vapor atmosférico producto de la evaporación de agua oceánica proveniente del Golfo de México son los alisios, los cuales son regulares durante todo el año.

Dichas características deben de permitir establecer una LAML) perfectamente representativa de su composición isotópica, en ausencia de perturbaciones debidas a (a) factores de fraccionamiento distintos al que es debido solamente a la altitud, ni (b) fuentes de agua distintas a las masas de vapor derivadas de evaporación marina en el Golfo de México.
A medida que la elevación topográfica se incrementa, la temperatura de las masas de aire reportadas y las precipitaciones disminuyen. De acuerdo con el Servicio Meteorológico Nacional (2012), existen variaciones climáticas contrastantes relacionadas con la elevación topográfica, teniendo un clima cálido sub-húmedo en la Planicie Costera del Golfo con temperaturas promedio anuales entre $22^{\circ}$ y $26^{\circ} \mathrm{C}$ y una precipitación promedio anual de $974.4 \mathrm{~mm}$. En el estado de Hidalgo, al centro de la sección, el clima es templado húmedo, con temperaturas de $18^{\circ}$ a $22^{\circ} \mathrm{C}$ y una precipitación promedio anual de $652.4 \mathrm{~mm}$. En el centro del país, en las inmediaciones de los estados de Querétaro e Hidalgo, el clima es templado sub-húmedo y presenta temperaturas de $18^{\circ}$ a 22 ${ }^{\circ} \mathrm{C}$ y una precipitación promedio anual de 589.9 $\mathrm{mm}$.

\section{Metodología}

La metodología llevada a cabo para cumplir los propósitos del trabajo se dividió en 3 etapas: análisis de relieve, muestreo de campo y análisis de laboratorio, que se detallan a continuación.

\subsection{ANÁLISIS DE RELIEVE}

Se desarrolló un análisis morfométrico del relieve con el software ArcGis versión 9.2, utilizando la topografía de 8 cartas a escala 1:50000 de INEGI en formato shapefile: F14C48, F14C49, F14C58, F14C59, F14D31, F14D32, F14D41 y F14D22. Se obtuvo, en primer lugar, un MDE (Modelo Digital de Elevación) que se reclasificó con la finalidad de obtener un mapa de elevaciones a la misma escala (Figura 4). El mapa de elevaciones obtenido a partir de un MDE muestra en colores cálidos las elevaciones topográficas más elevadas de la región, cambiando a tonos verdosos conforme disminuye la cota. Posteriormente, se determinó la sección de estudio en función del continuo decremento en la cota topográfica que se obtuvo en el mapa, eligiendo localidades para el mues- 


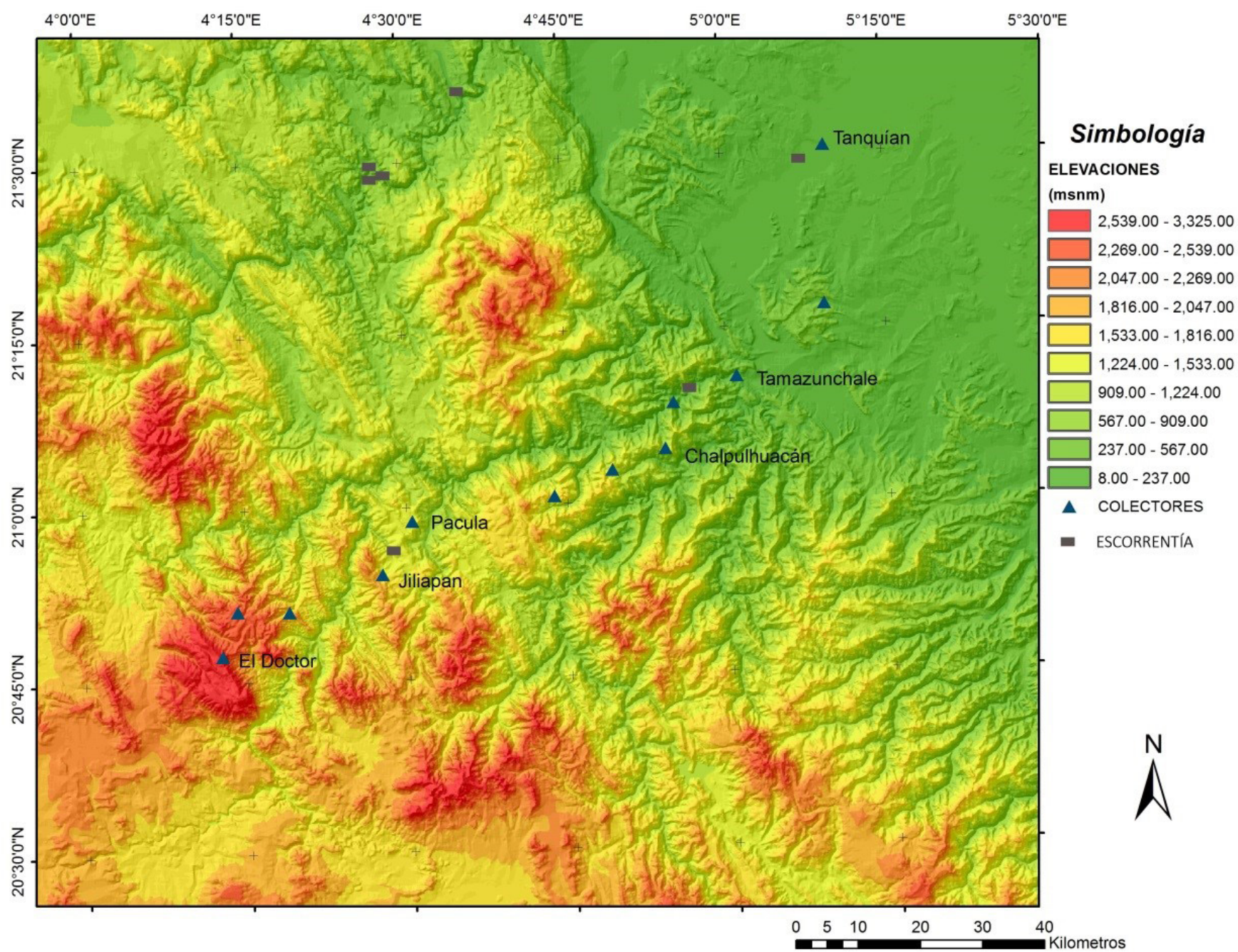

Figura 4 Mapa digital de elevaciones de la región estudiada: los tonos cálidos corresponden a las elevaciones mayores y los fríos a las de menor cota topográfica. La localización de los colectores de agua de lluvia instalados se indica con triángulos azules, los de escorrentía se indican en rectángulos ocres.

treo en las zonas topográficamente más altas que representaran la pendiente de la cuña orogénica. Se aprecia que las localidades donde se colocaron gradualmente disminuyen en elevación, siguiendo un relieve que decrece hacia el noreste (Figura 4). Con ello se evitó instalar colectores de agua pluvial en depresiones topográficas mostradas en el análisis de elevaciones; dichas depresiones representan valles o cuerpos de agua importantes, como lagos o lagunas. Igualmente, se pudieron evitar zonas con microclimas o evapotranspiración de zonas boscosas, favoreciendo con ello la captación es- pecíficamente de agua de lluvia no afectada por procesos secundarios de evaporación. Finalmente, se eligieron 12 localidades de muestreo, con elevación máxima de 2740 msnm y mínima de 56 msnm (ver Tabla 1).

\subsection{MUESTREO DE GAMPO}

Para realizar el muestreo de agua meteórica se construyeron 12 colectores de agua pluvial de tal forma que garantizaran el adecuado almacenaje del agua captada, teniendo particular cuidado 
en evitar la evaporación dentro del colector y el escape de vapor a la atmósfera. Para ello se utilizaron bidones de 20 litros de capacidad, forrados con materiales reflectantes (hacia el exterior) y aislantes térmicos (hacia el interior), como hule bajo-alfombra. Se colocó una cubierta reflectante de aluminio como recubrimiento final, con el propósito de evitar la radiación solar incidente. En la parte superior del bidón se colocó un embudo de 15 centímetros de diámetro para captar el agua pluvial, cubierto por una malla de alambre que sirvió como filtro de partículas con diámetros mayores a $2 \mathrm{~mm}$. Por último se agregó una capa de aproximadamente $3 \mathrm{~mm}$ de aceite mineral en el interior del bidón, a modo de tapón para evitar el escape de vapor que pudiera ser generado dentro del bidón.

Los colectores se instalaron en las localidades seleccionadas, en azoteas con espacios libres de cualquier escurrimiento y de acceso restringido (preferencialmente en edificios como escuelas o presidencias municipales) y sobre bases pintadas de blanco para evitar al máximo efectos indeseables debidos al calentamiento del piso por radiación solar. La época seleccionada para colectar el agua pluvial fue la temporada de lluvias del año 2012, entre los meses de julio y octubre, En el mismo periodo se tomaron muestras de agua de manantiales y ríos de flujo constante en 7 localidades ubicadas en las inmediaciones de los puntos de muestreo donde se instalaron los colectores, con la finalidad de obtener composiciones isotópicas de control en el sistema isotópico de la región (Figura 4). Durante la temporada de lluvia los colectores fueron constantemente monitoreados para evitar que se derramaran o contaminaran.

\subsection{ANÁLISIS ISOTÓPICOS}

Una vez concluida la temporada de lluvia se recuperaron intactos los colectores de los sitios donde se instalaron, pudiendo recuperar el $100 \%$ de muestra de agua en ellos, en condiciones óptimas para su análisis en laboratorio. El análisis de la composición isotópica de $\mathrm{H}$ y $\mathrm{O}$ se efectuó en el Laboratorio de Isótopos Estables del Instituto de
Geología de la UNAM. En primer lugar, se separaron las fases de aceite y sólidas de las muestras de agua mediante embudos de separación y papel filtro. Las muestras que contenían materia orgánica se agitaron con carbón activado para adsorberla y eliminarla de la fase líquida. Una vez obtenidos aproximadamente $100 \mathrm{~mL}$ de muestra de agua de cada bidón, se filtraron para eliminar cualquier residuo solido. Para las muestras tomadas de escorrentía se siguió el mismo procedimiento.

Los análisis de $\delta^{2} \mathrm{H}$ se efectuaron mediante un espectrómetro de absorción láser Los Gatos Research modelo 908-0008-3001, y para los análisis de $\delta^{18} \mathrm{O}$ se utilizó un espectrómetro de masas Thermo MAT 253 con una interfaz acoplada Thermo Finnigan de GASBENCH II. La calidad de los análisis de cada muestra fue controlada mediante el uso de los estándares de trabajo VSMOW, GISP, SLAP (ver Coplen, 1988) y agua tipo I FLEX 2010 grado HPLC. Los datos se reportaron mediante la notación convencional delta como desviaciones por mil respecto al estándar del promedio del agua oceánica (VSMOW, Vienna Standard Mean Oceanic Water).

\section{Resultados}

Los resultados de $\delta^{18} \mathrm{O}$ y $\delta^{2} \mathrm{H}$ obtenidos de agua pluvial y escorrentía se encuentran contenidos en la Tabla 1 y en las figuras 5 y 6 . La relación entre las composiciones isotópicas de las muestras de agua de lluvia y la elevación de las localidades estudiadas se muestra en las figuras 7 y 8 (para $\delta^{2} \mathrm{H}$ y $\delta^{18} \mathrm{O}$, respectivamente). Dicha relación es lineal e inversa respecto al incremento en la cota topográfica. El valor de $\mathrm{R}^{2}$ obtenido de $\delta^{2} \mathrm{H}$ y $\delta^{18} \mathrm{O}$ es de 0.90 y 0.91 , respectivamente.

Los valores de $\delta^{2} \mathrm{H}$ obtenidos para muestras de agua de lluvia fluctúan entre -80 y $-25 \%$ y los de $\delta^{18} \mathrm{O}$ varían entre -12 y $-4 \%$. Los valores de $\delta^{2} \mathrm{H}$ para las muestras de agua de escorrentía varían entre -60 y -36 \% y los de $\delta^{18} \mathrm{O}$ varían entre -10 y $-6 \%$. Estos valores, tanto de agua pluvial como de agua de escorrentía en la sección estudiada son 
Tabla 1. Relación de puntos de muestreo de agua, su localización geográfica de los mismos, y composición isotópica en $\mathrm{H}$ y $\mathrm{O}$ del agua respecto a VSMOW.

\begin{tabular}{|c|c|c|c|c|c|c|}
\hline Muestra & Localidad & Altura (msnm) & $\mathbf{X}(\mathbf{m})$ & $\mathbf{Y}(\mathbf{m})$ & $\delta^{2} H(\% 0)$ & $\delta^{18} \mathrm{O}(\%)$ \\
\hline \multicolumn{7}{|c|}{ Agua de lluvia: } \\
\hline TRA-12 & El Doctor & 2743 & 521439 & 2351174 & -80.34 & -11.75 \\
\hline TRA-11 & San Joaquín & 2430 & 511266 & 2346815 & -68.87 & -10.28 \\
\hline TRA-10 & Apartadero & 1640 & 510007 & 2339356 & -60.06 & -9.05 \\
\hline TRA-9 & Jiliapan & 1525 & 501441 & 2335912 & -52.42 & -8.33 \\
\hline TRA-8 & Pacula & 1313 & 492144 & 2331613 & -57.18 & -8.84 \\
\hline TRA-7 & Cerro Prieto & 1589 & 469229 & 2327523 & -48.15 & -8.01 \\
\hline TRA-6 & Santa Ana de Allende & 1387 & 464454 & 2318887 & -42.09 & -7.03 \\
\hline TRA-5 & Chapulhuacán & 961 & 449449 & 2312756 & -42.83 & -7.15 \\
\hline TRA-4 & Taman (Los Amigos) & 390 & 441149 & 2312756 & -32.55 & -5.91 \\
\hline TRA-3 & Tamazunchale & 153 & 438715 & 2305551 & -36.72 & -6.38 \\
\hline TRA-2 & San Martín Chalchicuautla & 201 & 425475 & 2294723 & -30.76 & -5.63 \\
\hline TRA-1 & Tanquián & 56 & 401437 & 2292969 & -25.35 & -4.89 \\
\hline \multicolumn{7}{|c|}{ Escorrentía: } \\
\hline \multicolumn{7}{|c|}{ Agua de río } \\
\hline E-1 & San Martín Chalchicuautla & 282 & 506912 & 2370178 & -31 & -5.71 \\
\hline E-2 & Xilitla & 694 & 500914 & 2364554 & -35.8 & -6.28 \\
\hline E-3 & Xilitla & 696 & 500914 & 2364554 & -35.6 & -6.25 \\
\hline E-4 & Pacula & 1818 & 466176 & 2321347 & -53.3 & -8.04 \\
\hline E-5 & Apartadero & 1865 & 446306 & 2314238 & -64.5 & -9.79 \\
\hline \multicolumn{7}{|c|}{ Agua de manantiales y cuevas } \\
\hline E-6 & Apartadero & 1885 & 446306 & 2314238 & -63.3 & -9.66 \\
\hline E-7 & Xilitla & 529 & 501012 & 2365438 & -34.5 & -6.19 \\
\hline
\end{tabular}

Nota: la altura y coordenadas utilizadas fueron las registradas por medio de GPS, con datum WGS84, zona 14.

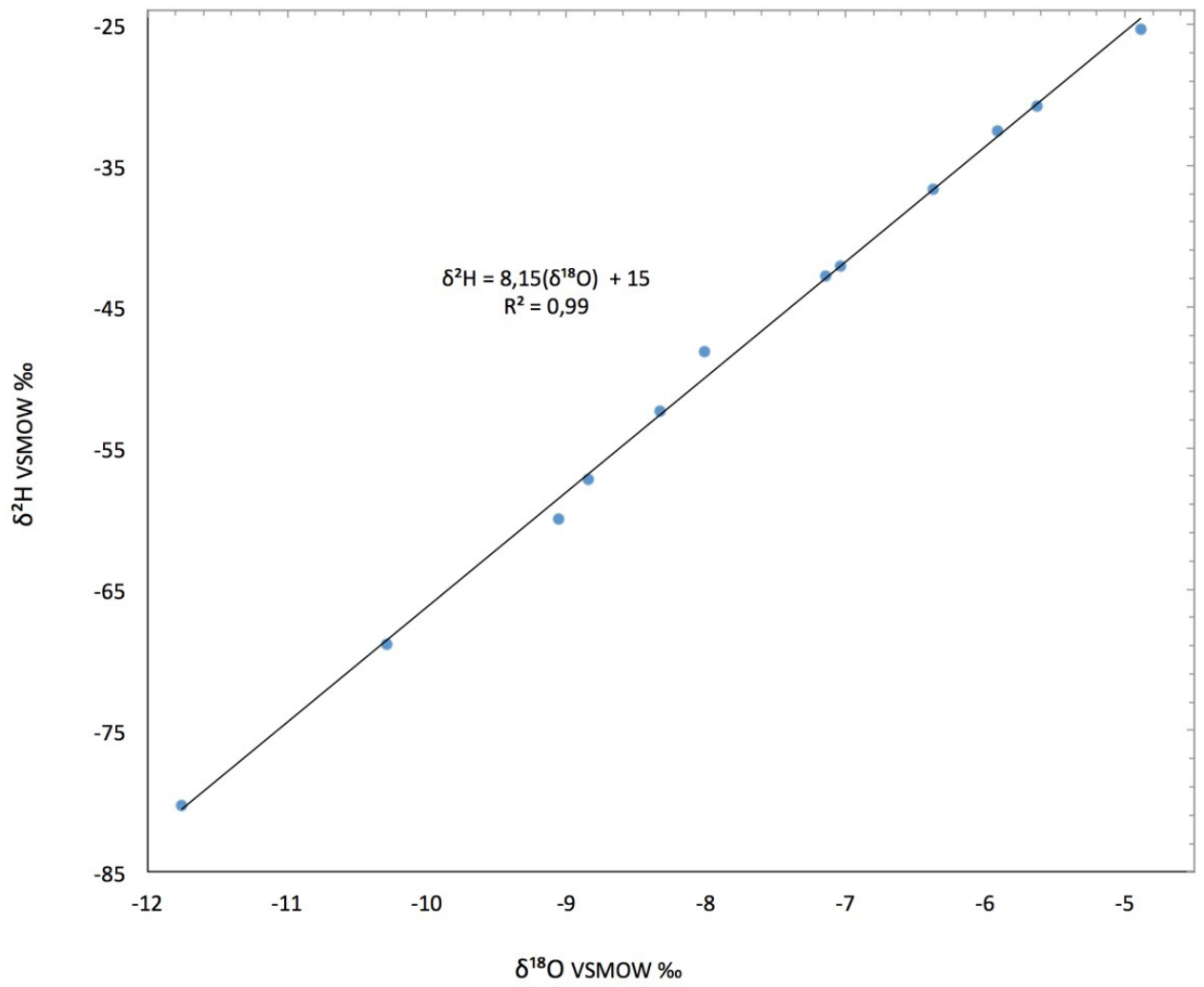

Figura 5 Línea de Agua Meteórica Local obtenida por el análisis de 12 colectores de agua pluvial dentro de la zona estudiada. 


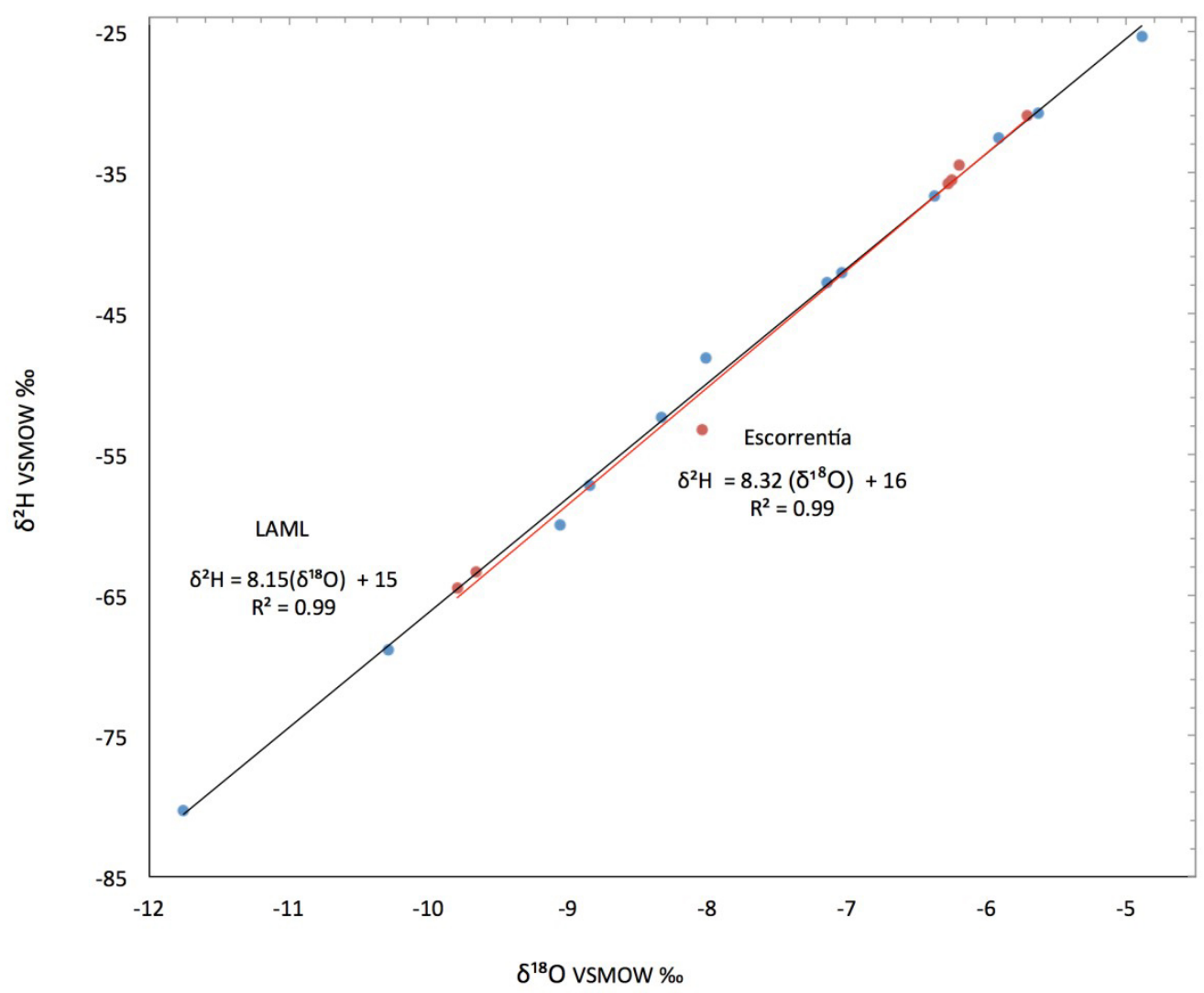

Figura 6 Comparativa de la Línea de Agua Meteórica Local en negro y Línea de Agua Meteórica de escorrentía en rojo, esta muestra la diferencia entre las ecuaciones de la recta que definen a ambas.

progresivamente menores a medida que se incrementa la elevación topográfica (Tabla 1, figuras 9 y 10). Cabe hacer notar que el agua de escorrentía presenta composiciones isotópicas similares a la del agua de lluvia en muestras obtenidas a elevaciones topográficas similares (Figura 6).

Con los resultados obtenidos de las concentraciones isotópicas en agua de lluvia se definió la ecuación de regresión lineal que representa la LAML, que define la variación de $\delta^{2} \mathrm{H}$ y $\delta^{18} \mathrm{O}$ (Figura 5) del agua meteórica a lo largo de la sección estudiada en el Cinturón de Pliegues y Cabalgaduras Mexicano. La ecuación de la recta que la define es:

$$
\delta^{2} \mathrm{H}=\delta^{18} \mathrm{O} * 8.15+15
$$

Ésta es similar a la Línea de Agua Meteórica Mundial (Craig, 1961):

$$
\delta^{2} \mathrm{H}=\delta^{18} \mathrm{O} * 8+10
$$

La diferencia de la ordenada al origen que exhibe la LAML respecto a la línea de agua meteórica co- rrespondiente al promedio mundial definido por Craig (1961) es de +5; el valor de $\mathrm{R}^{2}$ obtenido en la LAML es de 0.99, mientras que la variación en la pendiente es de 0.15 .

En comparación, la Línea de Agua Meteórica que se obtiene del análisis de agua de escorrentía se define por la ecuación:

$$
\delta^{2} \mathrm{H}=\delta^{18} \mathrm{O} * 8.32+16
$$

La diferencia de la ordenada al origen que exhibe dicha correlación lineal respecto a la LAML definida en el presente estudio es de +1 ; el valor de $\mathrm{R}^{2}$ es de 0.99 , mientras que la variación en la pendiente es de 0.17 respecto a la misma (Figura 6).

La tasa de fraccionamiento isotópico calculada por mínimos cuadrados por cada kilómetro de elevación obtenido a partir de los valores de muestras de agua de lluvia es de $-2.19 \% \mathrm{~km}^{-1}$ para $\delta^{18} \mathrm{O}$ y de $-17.75 \% \mathrm{~km}^{-1}$ para $\delta^{2} \mathrm{H}$ (figuras 9 y 10 ). 

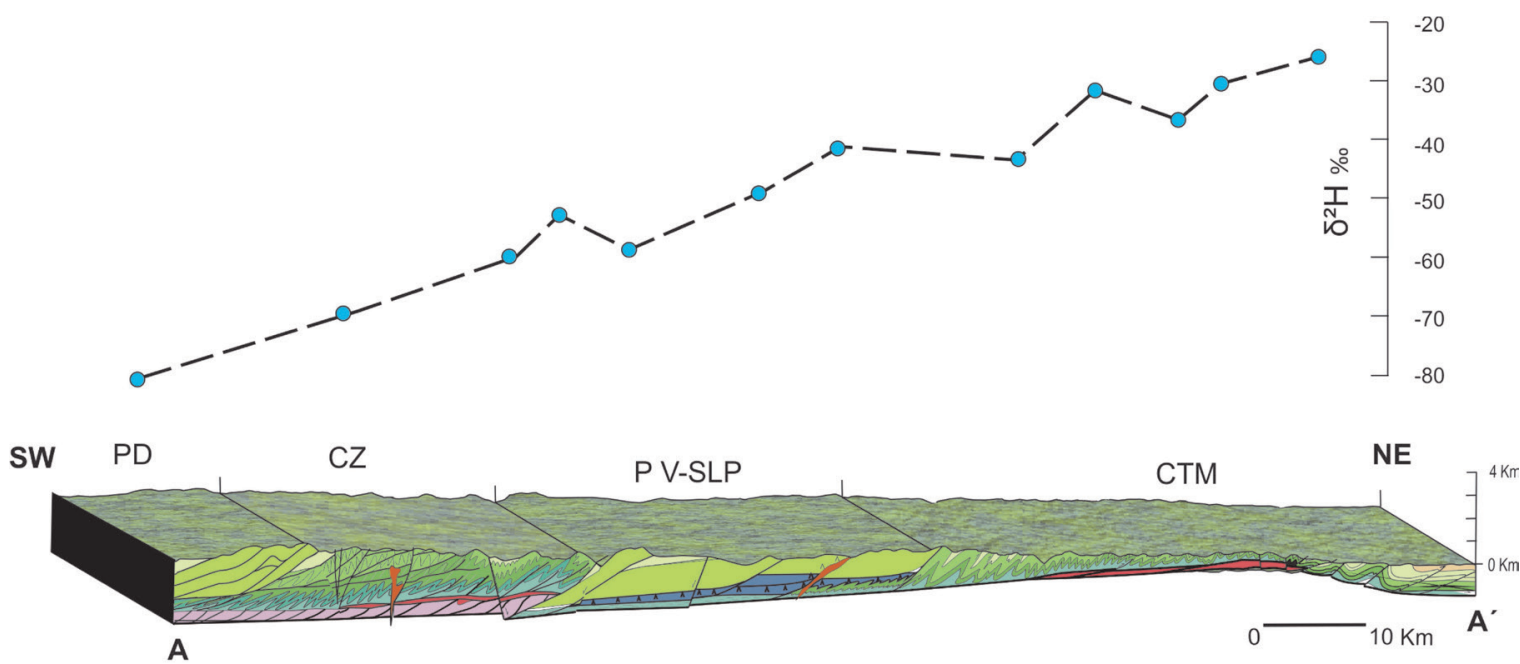

Figura 7 Variaciones en los valores de la composición isotópica de $\mathrm{H}$ en el perfil geológico y topográfico de la sección estudiada. Clave: PD = Plataforma El Doctor; CZ = Cuenca de Zimapán; PV-SLP = Plataforma Valles-San Luis Potosí; CTM = Cuenca Tampico-Misantla.

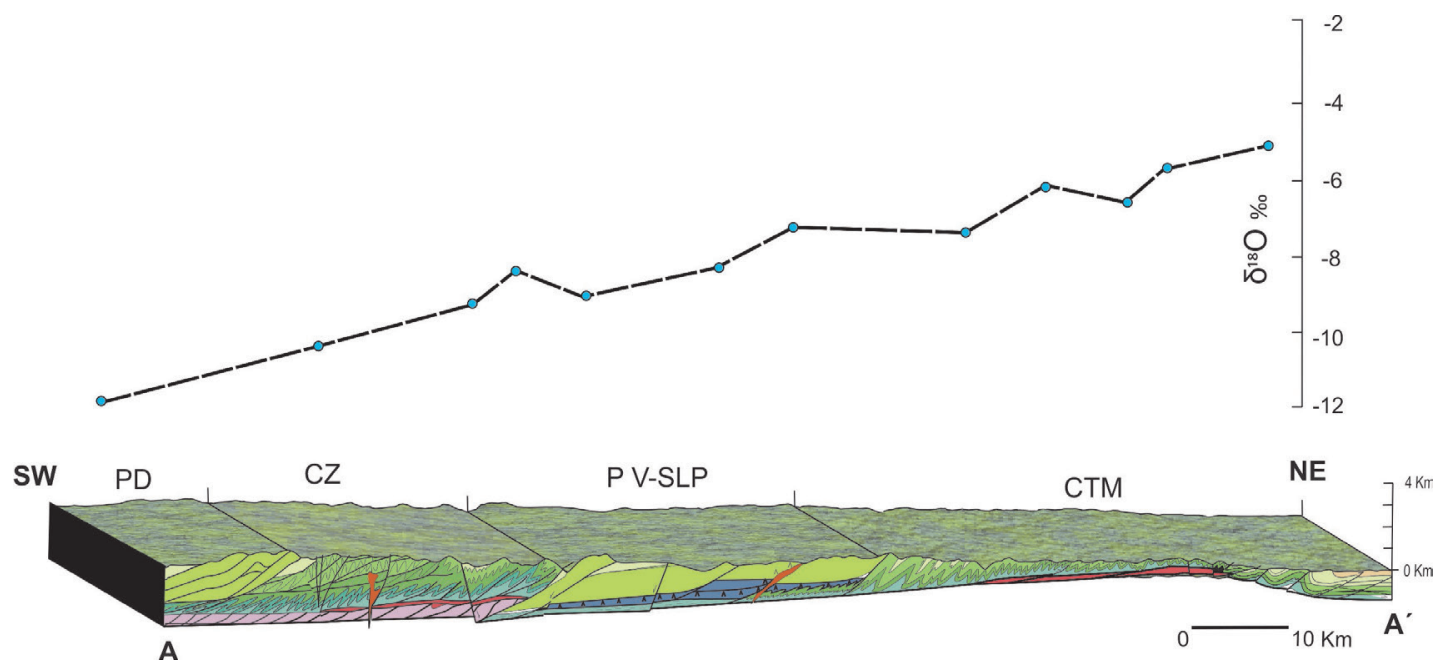

Figura 8 Variaciones en los valores de la composición isotópica de $\mathrm{O}$ en el perfil geológico y topográfico de la sección estudiada. Clave: PD = Plataforma El Doctor; CZ = Cuenca de Zimapán; PV-SLP = Plataforma Valles-San Luis Potosí; CTM = Cuenca Tampico-Misantla.

\section{Discusión}

La sección elegida para el análisis de la variación en la composición isotópica de agua meteórica en este trabajo forma parte de una porción central del Cinturón de Pliegues y Cabalgaduras Mexicano, el cual tiene un rasgo morfológico de cuña orogénica que decrece en elevación topográfica de manera regular al noreste del país (Figura 3). La zona de estudio presenta una pendiente prácticamente constante, con diferencias de latitud muy escasas y en ausencia de barreras orográficas relevantes, a diferencia de otros sitios en el mundo donde se han realizado estudios con propósitos similares, tales como la Sierra Nevada en la Alta California (Ingraham y Taylor, 1991), los Alpes (Campani et al., 2012), los Táurides en Turquía (Schemmel et al., 2013), o una sección que parte del Citlaltépetl hasta la planicie del Golfo de México (Pérez Quezadas et al., 2015), entre otros. Las ventajas de 


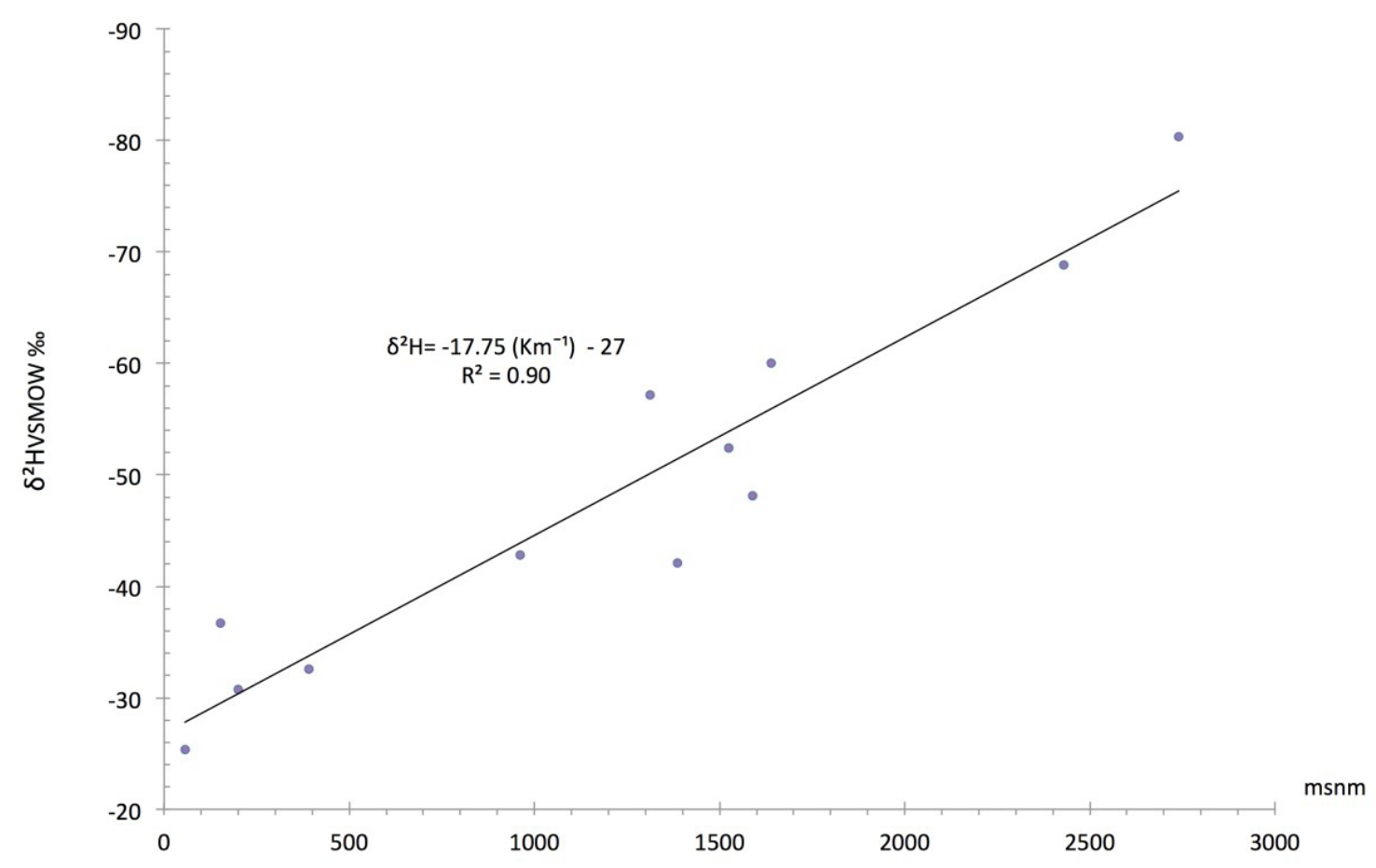

Figura 9 Correlación entre la composición isotópica de $\mathrm{H}$ obtenida en el análisis del agua de lluvia de la sección estudiada respecto a las variaciones de altitud.

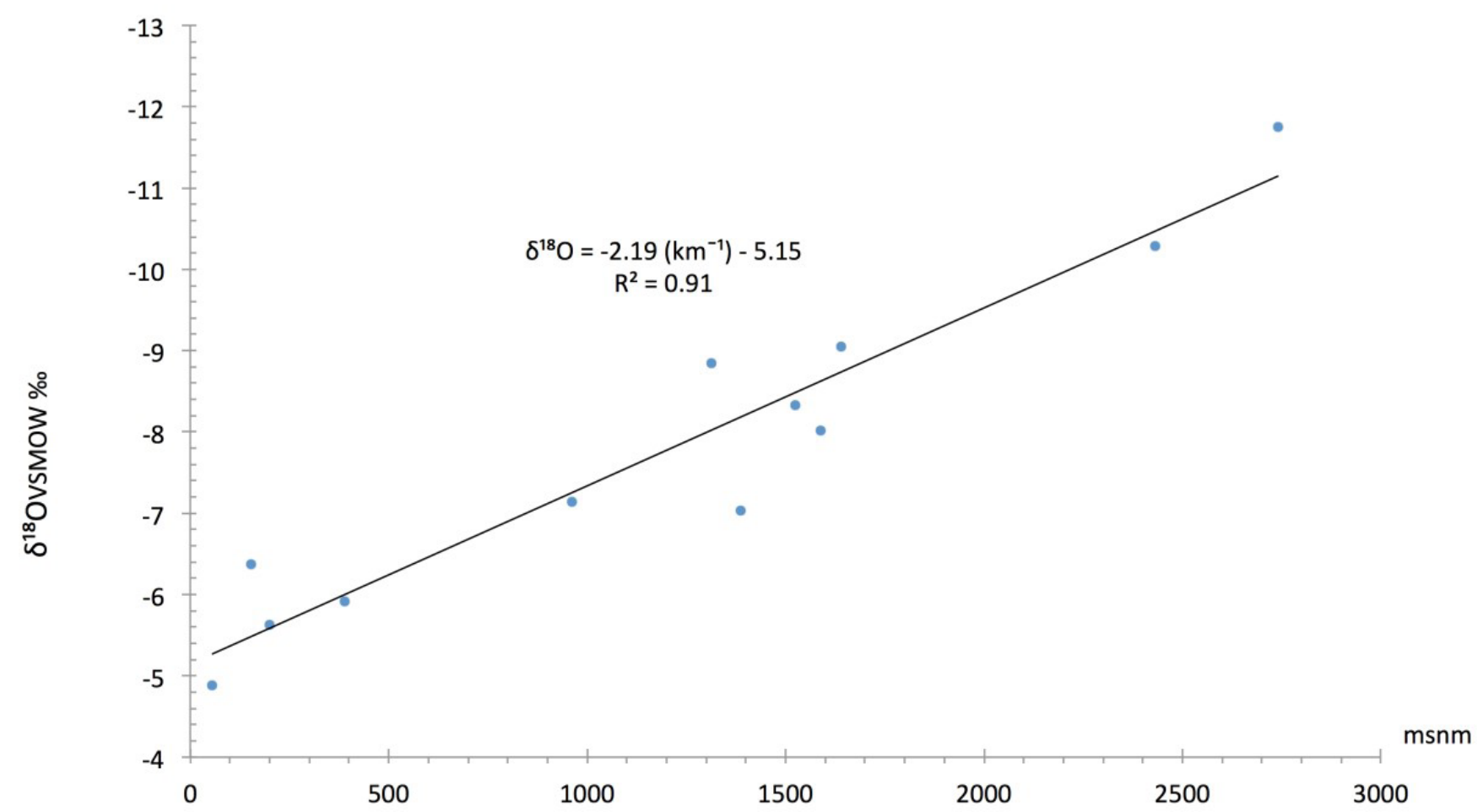

Figura 10 Correlación entre la composición isotópica de $\mathrm{H}$ obtenida en el análisis del agua de lluvia de la sección estudiada respecto a las variaciones de altitud. 
contar con una sección de estudio como la utilizada en este trabajo son múltiples, pues ello permite (1) calcular la tasa de fraccionamiento en función de la altura con mucha mayor precisión que en los casos antes mencionados, y (2) eliminar los efectos de los diversos factores de fraccionamiento adicionales al que es exclusivamente debido a la elevación topográfica.

Los valores que se obtuvieron durante el análisis de la composición isotópica de agua meteórica de la temporada de lluvias de 2012 muestran valores de composiciones isotópicas progresivamente menores conforme se incrementa la elevación topográfica (figuras 7 y 8). Dicha tendencia ha sido discutida en diversos estudios, destacando el de Poage y Chamberlain (2001), quienes compilaron datos de 68 regiones del mundo ubicadas en Norteamérica, Centroamérica, Sudamérica, Europa, el Himalaya (hasta los $5000 \mathrm{msnm}$ ) y localidades en extremos latitudinales terrestres norte y sur. Poage y Chamberlain (2001) reportan la misma tendencia en el empobrecimiento de isótopos pesados de agua meteórica en todos los casos estudiados, debido a que el vapor atmosférico que se interna al continente es afectado por la destilación tipo Rayleigh y la obstrucción de barreras topográficas.

La relación entre $\delta^{2} \mathrm{H}$ y $\delta^{18} \mathrm{O}$ para el agua meteórica es proporcional y fue definida por Craig (1961) como la Línea de Agua Meteórica Mundial (LAMM), en un estudio que integra datos de diversas partes del mundo. En éste se considera la posición latitudinal de algunas regiones como principal factor de empobrecimiento o enriquecimiento de isótopos pesados. Con base en dicha observación y a lo discutido en trabajos posteriores (v.g., Friedman et al., 1964; Mook, 2002; Sharp, 2007) se puede afirmar que, en la sección objeto del presente estudio, la posición latitudinal controla la temperatura que incide sobre la superficie oceánica, siendo éste el primer factor de fraccionamiento isotópico hacia la fase de vapor. Lo anterior se encuentra representado por el valor de la ordenada al origen de la ecuación de la LAML que es +15 en las muestras colectadas con agua de lluvia, respecto al de la LAMM que es +10 , así como la ligera desviación en la pendiente, de 8.15 , respecto al valor del promedio global, que es de 8 . Ello refleja condiciones de mayor evaporación al promedio mundial en la latitud comprendida entre $\operatorname{los} 21^{\circ}$ y $22^{\circ}$ norte, representada por la sección orogénica estudiada. El valor de $\mathrm{R}^{2}$ de la LAML es de 0.99, lo que indica un buen ajuste hacia la recta, ocasionado por la adecuada captura in situ, la ausencia de fuentes de vapor atmosférico residual en el muestreo y el correcto proceso de análisis de agua de lluvia. Por otra parte, las muestras analizadas de agua de escorrentía forman parte del mismo sistema isotópico y no parecen tener una fuente secundaria que aporte agua a ríos y manantiales, reflejado en la ecuación lineal que la define (Ecuación 3) la cual muestra una ligera desviación en la pendiente (de 8.32), así como en la ordenada al origen $(+16)$ respecto a la LAML (Figura 6). Esto es un comportamiento esperable originado por el transporte que ha tenido sobre la superficie terrestre y la evaporación que se origina como consecuencia de ello.

En comparación, en un estudio similar en la Sierra Nevada de la Alta California, Ingraham y Taylor (1991) reportaron tasas de fraccionamiento en función del incremento en la cota topográfica para isótopos de $\mathrm{O}$ entre -3.1 y $-2.1 \% \mathrm{~km}^{-1}$, correspondientes respectivamente a latitudes entre $37^{\circ}$ y $39^{\circ}$ N. En cambio, a latitudes entre $33^{\circ}$ y $37^{\circ} \mathrm{S}$, Rozanski y Araguás (1995) obtuvieron tasas de fraccionamiento de isótopos de $\mathrm{O}$ entre -2.1 y $-2.2 \% \mathrm{~km}^{-1}$. Las diferencias en el valor de las tasas de fraccionamiento isotópico reportados en dichos trabajos no son ocasionadas sustancialmente por la posición latitudinal, de lo cual es posible deducir que el empobrecimiento en isótopos pesados en las secciones anteriormente mencionadas es más sensible a cambios en el incremento de la topografía y a la obstrucción propiciada por las barreras orogénicas que a otros factores de fraccionamiento. La pendiente regular del área de estudio propicia la entrada homogénea de vapor atmosférico derivado de la evaporación de agua de mar, por medio de los vientos alisios que entran por el Golfo de 
México. Como consecuencia de ello, el valor de $\mathrm{R}^{2}$ (de 0.99) de la LAML ajusta a la recta, lo que indica ausencia de fuentes secundarias de vapor que modificarían la composición isotópica de la fuente primordial. Poage y Chamberlain (2001) y Schemmel et al. (2013) discuten la participación de barreras topográficas y diversas masas de aire entrando en una cordillera orogénica como factores principales que modifican la composición isotópica. Schemmel et al. (2013) determinaron tres Líneas de Agua Meteórica Local en distintas secciones estudiadas en las montañas Taurus y Pontiac en Turquía, con diferencias considerables respecto a la LAMM obtenida por Craig (1961):

LAML Montañas Pontiac: $\delta^{2} \mathrm{H}=\delta^{18} \mathrm{O} * 7.1+7.3$

LAML Montañas Taurus: $\delta^{2} \mathrm{H}=\delta^{18} \mathrm{O} * 7.2+10.8$

LAML Valle interior: $\delta^{2} \mathrm{H}=\delta^{18} \mathrm{O} * 4.0-29.3$

Las ecuaciones 4 a 6 (Schemmel et al., 2013) muestran diferencias sustanciales entre sí, tanto en la pendiente como en la ordenada al origen. En las ecuaciones 4 y 5 no existe una fuente secundaria de vapor que modifique la concentración de isótopos de agua meteórica. Sin embargo, en la ecuación 6, determinada en el valle formado por ambas cordilleras, existe una notoria aportación de vapor originado por procesos secundarios, lo cual puede identificarse tanto en la pendiente como en la ordenada al origen. Así, en comparación, la similitud entre la LAML obtenida para este trabajo (Ecuación 1) respecto a las ecuaciones 2 (LAMM de Craig, 1961), 4 y 5 (Schemmel et al., 2013) sugiere la ausencia de fuentes secundarias de vapor, lo cual indica que el vapor del que derivaron las precipitaciones analizadas en este estudio es debido exclusivamente a la evaporación de agua marina en el Golfo de México.

Las variaciones en los valores de $\delta^{2} \mathrm{H}$ y $\delta^{18} \mathrm{O}$ establecen, en ambos casos, correlaciones lineales con la elevación topográfica, de modo que ésta constituye el factor de fraccionamiento esencial para la sección estudiada. Este resultado era el esperado por el modo en que fue seleccionada la zona de estudio y en que fue diseñado el muestreo. Las tasas de fraccionamiento obtenidas en el presente estudio son debidas a la pendiente continua que permite llevar a cabo la destilación Rayleigh de manera ideal y sin barreras que afecten dicho proceso. La buena correlación ente valores isotópicos y altitud obtenida indica que no existen algunas otras fuentes de vapor residual (e.g., concentrado por algún alto topográfico) las cuales pudieran modificar el valor de la composición isotópica en la región. Es decir, el valor de las tasas de fraccionamiento isotópico para $\mathrm{H}$ y $\mathrm{O}$ analizadas en este trabajo sólo es debido al incremento de la elevación topográfica.

\section{Conclusiones}

En este trabajo se determinaron las variaciones en $\delta^{2} \mathrm{H}_{\text {vSMOw }}$ y $\delta^{18} \mathrm{O}_{\text {vSMOw }}$ y el fraccionamiento isotópico en el agua meteórica a lo largo de una sección del Cinturón de Pliegues y Cabalgaduras Mexicano (CPGM) con características óptimas para ello. Los resultados obtenidos reflejan un descenso en $\delta^{2} \mathrm{H}$ y $\delta^{18} \mathrm{O}$ a medida que la elevación topográfica aumenta, desde $\delta^{2} \mathrm{H}=-25.35 \%$ y $\delta^{18} \mathrm{O}=-4.89 \%$ en la cota topográfica más baja (56 msnm), hasta $\delta^{2} \mathrm{H}=-80.34 \%$ y $\delta^{18} \mathrm{O}=-11.75 \%$ en la cota topográfica más alta (2740 msnm).

La ecuación de la recta que define la Línea del Agua Meteórica Local (LAML; $\delta^{2} \mathrm{H}=\delta^{18} \mathrm{O} * 8.15$ +15 ) tiene una ordenada al origen de 15 , a diferencia del promedio mundial definido en la Línea del Agua Meteórica Mundial, con un origen de 10. Ello es debido al efecto de una mayor evaporación a los $21^{\circ} \mathrm{N}$ en el Golfo de México que en el promedio global.

Las tasas de fraccionamiento en función de la elevación topográfica que se obtuvieron en la sección estudiada fueron de $-2.19 \% \mathrm{~km}^{-1}$ para $\delta^{18} \mathrm{O}$, y de $-17.75 \% \mathrm{~km}^{-1}$ para $\delta^{2} \mathrm{H}$.

La ecuación de la recta que define la Línea de Agua Meteórica en agua de escorrentía muestra una ligera variación respecto a la LAML, tanto en la pendiente, como en la ordenada al origen, lo cual es consecuencia de la evaporación que ha sufrido durante su recorrido desde la zona de recarga, hacia el punto donde se colectó. 


\section{Agradecimientos}

Los autores agradecemos de manera especial a los revisores Elena Lounejeva y Pura Alfonso, quienes contribuyeron a mejorar este trabajo, también queremos agradecer a Francisco Otero y Rafael Puente del Laboratorio de Isótopos Estables del Instituto de Geología de la UNAM por la ayuda brindada durante el procesamiento y análisis de las muestras. Esta investigación fue financiada por el proyecto de CONACYT número 155662 y por presupuesto del Instituto de Geología de la UNAM.

\section{Referencias}

Ambach, W., Dansgaard, W., Eisner, H., Moller, J., 1968, The altitude effect on the isotopic composition of precipitation and glacier ice in the Alps: Tellus XX, 4, 595-598.

Birkle, P., Marín, E.P., Pinti, D.L., Castro, M.C., 2016, Origin and evolution of geothermal fluids from Las Tres Vírgenes and Cerro Prieto fields, Mexico - Co-genetic volcanic activity and paleoclimatic constraints: Applied Geochemistry, 65, 36-53.

Campa-Uranga, M.F. 1983, The tectonostratigraphic terranes and the thrust belt in Mexican territory: Stanford University Publications, Geological Sciences, 18, 44-46.

Campani, M., Mulch, A., Kempf, O., Schlunegger, F., Mancktelow, N., 2012, Miocene paleotopography Central Alps: Earth and Planetary Science Letters, 337, 174-185.

Carrillo, B.J., 1971, La Plataforma de VallesSan Luis Potosí: Boletín de la Asociación Mexicana de Geólogos Petroleros, 22, 1-102.

Carrillo-Martínez, M., 1990, Geometría estructural de la Sierra Madre Oriental, entre Peñamiller y Jalpan, Estado de Querétaro: Revista Mexicana de Ciencias Geológicas, 9, 62-70.
Chamberlain, C.P., Poage, M.A., Craw, D., Reynolds, R.C., 1999, Topographic development of the South Alps recorded by the isotopic composition of authigenic clay minerals. South Island, New Zealand: Chemical Geology, 155, 297-294.

Coplen, T., 1988, Normalization of oxygen and hydrogen isotope data: Chemical Geology, 72, 273-297.

Craig, H., 1961, Isotopic variations in meteoric waters: Science, 133, 1702-1703.

Dansgaard, W., 1964, Stable isotopes in precipitation: Tellus, 16, 436-468.

Davis, D.M.; Suppe, J., Dahlem, F.A., 1983, Mechanics of fold-and- thrust belts and acretionary wedges: Journal of Geophysical Research, 88(1), 153-172.

Eguiluz de Antuñano, S., Aranda-Gómez, M., Marret, R., 2000, Tectónica de la Sierra Madre Oriental, México: Boletín de la Sociedad Geológica Mexicana, 53, 1-26.

Fitz-Díaz, E., Hudleston, P., Tolson, G., 2010, Comparison of tectonic styles in the Mexican and Canadian Rocky Mountain Fold-Thrust Belt: The Geological Society of London, Special publications, 349, 149-167.

Fitz-Díaz, E., Hudleston, P., Siebenaller, L., Kirschner, D., Camprubí, A., Tolson, G., Pi Puig, T., 2011, Insights into fluid and water-rock interaction during deformation of carbonate sequences in the Mexican foldthrust belt: Journal of Structural Geology, 33, 1237-1253.

Fitz-Díaz, E., Camprubí, A., CienfuegosAlvarado, E., Morales-Puente, P., Schleicher, A., van der Pluijm, B., 2014, Newly-formed illite preserves fluid sources during folding of shale and limestone rocks; an example from the Mexican Fold-Thrust Belt: Earth and Planetary Science Letters, 391, 263-273.

Friedman, I., Redfield, A.C., Schoen, B., Harris, J., 1964, The variation of the deuterium content of natural waters in the hydrologic cycle: Reviews in Geophysics, 2, 177-224. 
Garzione, C.N., Dettman, D.L., Quade, J., DeCelles, P.G., Butler, R.F., 2000, High times on the Tibetan Plateau: Paleoelevation of the Thakkhola graben, Nepal: Geology, 28, 339-342.

González-Partida, E., Carrillo-Chávez, A., Levresse, G., Tello-Hinojosa, E., VenegasSalgado, S., Ramírez-Silva, G., Pal-Verma, M., Tritlla, J., Camprubí, A., 2005, Hydrogeochemistry and isotopic fluid evolution of the Los Azufres geothermal field, Central Mexico: Applied Geochemistry, 20, 23-39.

Ingraham, N.L., Taylor, B.E., 1991, Light stable isotope systematics of large-scale hydrologic regimes in California and Nevada: Water Resources Research, 27, 77-90.

Molnar, P., 2010, Deuterium and oxygen isotopes, paleoelevations of the Sierra Nevada, and Cenozoic climate: Geological Society of America Bulletin, 122, 1106-1115.

Mook, W.G., 2002, Isótopos ambientales en el ciclo hidrológico, principios y aplicaciones: Publicaciones del Instituto Geológico y Minero de España, Serie: Guías y manuales, 1, $595 \mathrm{p}$.

Moser, H., Stichler, W., 1974, Deuterium and oxygen-18 contents as an index of the properties of snow covers: International Association of Hydrological Sciences Publication, 114, 122-135.

Ortega-Flores, B., 2011, Deformación por acortamiento en la Plataforma Valles-San Luis Potosí y en la Cuenca Tampico-Misantla; porción externa del Cinturón de Pliegues y Cabalgaduras Mexicano: Ciudad de México, México, Universidad Nacional Autónoma de México, Tesis de Maestría, 101 p.

Pérez Quezadas, J., Cortés Silva, A., Inguaggiato, S., Salas Ortega, M.R., Cervantes Pérez, J., Heilweil, V.M., 2015, Meteoric isotopic gradient on the windward side of the Sierra Madre Oriental area, Veracruz - Mexico: Geofísica Internacional, 54, 267-276.

Poage, M., Chamberlain, C., 2001, Empirical relationships between elevation and the stable isotope composition of precipitation and surfacewaters, Considerations for studies of paleoelevation change: American Journal of Science, 301, 1-15.

Poulsen, J.C., Jeffery, L.M., 2012, Climate change imprinting on stable isotopic compositions of high-elevation meteoric water cloaks past surface elevations of major orogens: Geology, 39, 595-598.

Rowley, D.B., 2007, Stable isotope-based paleoaltimetry: Theory and validation. Reviews in Mineralogy and Geochemistry, 66, 23-52.

Rowley, D.B., Garzione, C., 2007, Stable isotopebased Paleoaltimetry, Annual Reviews of Earth and Planetary Sciences, 35, 463-508.

Rozanski, K., Araguás-Araguás, L., 1995, Spatial and temporal variability of stable isotope composition of precipitation over the South American continent: Bulletin de l'Institut Français d'Études Andines, 24, 379-390.

Schemmel, F., Mikes, T., Rojay, B., Mulch, A., 2013, The impact of topography on isotopes in precipitation across the Central Anatolian Plateau (Turkey): American Journal of Science, 313, 61-80.

Suter, M., 1980, Tectonics of the external part of the Sierra Madre Oriental foreland thrust and fold belt Between Xilitla and the Moctezuma River (Hidalgo and San Luis Potosí states): Revista Mexicana de Ciencias Geológicas, 4, 19-31.

Suter, M., 1984, Cordilleran deformation along the eastern edge of the Valles-San Luis Potosi carbonate platform, Sierra Madre Oriental thrust and fold belt, east-central Mexico: Geological Society of America Bulletin, 95, 1387-1397.

Suter, M., 1987, Structural traverse across the Sierra Madre Oriental fold-thrust belt in east-central Mexico: Geological Society of America Bulletin, 98, 249-264.

Servicio Meteorológico Nacional, 2012, El Clima en México: Reporte del Clima en México 2012, 23 p. 
Sharp, Z., 2007, Principles of Stable Isotope Geochemistry, Prentice Hall, 334 p.

Tello, H.F., 1982, Características geoquímicas e isotópicas de los fluidos producidos por los pozos Los Humeros, Puebla, México: Geotermia, 8, 3-48.
Truesdell, A.H., Rye, R.O., Pearson, FJ., Olson, E.R., Nehring, N.L., Huebner, M., Coplen, T.B., 1978, Preliminary isotopic studies of fluids from the Cerro Prieto geothermal field: Proceedings $1^{\text {st }}$ Symposium on the Cerro Prieto, B.C., Mexico, Geothermal Field, 95-99. 